\title{
Competing chiral and multipolar electric phases in the extended Falicov-Kimball model
}

\author{
B. Zenker and H. Fehske \\ Institut für Physik, Ernst-Moritz-Arndt-Universität Greifswald, D-17489 Greifswald, Germany \\ C. D. Batista \\ Theoretical Division, Los Alamos National Laboratory, Los Alamos, New Mexico 87545, USA
}

(Dated: November 27, 2018)

\begin{abstract}
We study the effects of interband hybridization within the framework of an extended FalicovKimball model with itinerant $c$ and $f$ electrons. An explicit interband hybridization breaks the $\mathrm{U}(1)$ symmetry associated with the conservation of the difference between the total number of particles in each band. As a result, the degeneracy between multipolar electric and chiral orderings is lifted. We analyze the weak- and strong-coupling limits of the $c$ - $f$ electron Coulomb interaction at zero temperature, and derive the corresponding mean-field quantum phase diagrams at half-filling for a model defined on a square lattice.

PACS numbers: 71.10.Fd, 71.10.Hf, 71.28.+d, 71.35.-y
\end{abstract}

\section{INTRODUCTION}

The Falicov-Kimball mode ${ }^{1,2}, 2$ (FKM) was primarily introduced to describe the metal-insulator transition of the mixed-valence compound $\mathrm{SmB}_{6}$. Later on, the model became widely accepted as a minimal Hamiltonian for studying several strongly correlated electron systems,,$\underline{3-\underline{8}}$ in particular, heavy fermion compounds $\underline{\underline{9}} \underline{-11}$ In its original form, the FKM contains an itinerant $c$ band of electrons that interact via a local Coulomb repulsion with localized $f$ electrons. The spin degree of freedom of the electrons is not included. The local $f$ electron number is strictly conserved and $c$ - $f$ electron coherence cannot be established $\stackrel{12}{ }$ An explicit hybridization between $f$ and $c$ orbitals provides an opportunity to overcome this shortcoming $\underline{13,14}$ More recently, it was shown that a finite $f$ electron bandwidth also induces $c$ - $f$ electron coherence, i.e., it can lead to an excitonic condensate even in absence of an explicit interband hybridization. $\underline{\underline{15}, 16}$

These extended versions of the FKM were used to substantiate the exciting idea of electronic ferroelectricity (EFE) $\underline{14}-18$ The ferroelectric phase only appears when the $c$ and $f$ orbitals have opposite parity under spatial inversion. The concomitant spontaneous breaking of inversion symmetry results from a nonvanishing average of $\left\langle c^{\dagger} f\right\rangle$. Since this expectation value corresponds to (excitonic) pairing of electrons and holes from different bands, the appearance of EFE is directly related with the formation of an excitonic insulator (EI) $\frac{19-21}{2}$

The FKM with two dispersive bands, the so-called extended Falicov-Kimball model (EFKM), was studied previously for describing different properties of the EI phase $22-26$ However, as it was shown for the case of opposite-parity orbitals $\stackrel{15,16}{=}$ the inclusion of a finite interband hybridization can be very relevant because it removes the U(1) symmetry associated with the conservation of the difference between the total number of particles in each band: $N_{c}-N_{f}$. In particular, this hybridiza- tion term is certainly relevant when the ground-state of the EFKM corresponds to an excitonic condensate. For the EFKM with interband hybridization (HEFKM), the excitonic condensate is in general replaced by Ising-type phases that only break discrete symmetries of the Hamiltonian.

In this paper we present a mean-field study of the influence of an explicit hybridization on the symmetry-broken states that can take place for the HEFKM. To determine the ground-state quantum phase diagram of the HEFKM in the strong- and weak-coupling limits of $c$ - $f$ electron interaction, we assume that the interband hybridization amplitudes are small compared to the intraband hopping (transfer) integrals. Given the nature of the discrete symmetries of the HEFKM, the natural ground-state candidates are chiral phases (CHPs) and states with multipolar electric orderings.

\section{MODEL}

By expressing the orbital flavor as a pseudospin variable, $c_{i}^{\dagger} \equiv c_{i \uparrow}^{\dagger}$ and $f_{i}^{\dagger} \equiv c_{i \downarrow}^{\dagger}$, the Hamiltonian takes the form

$$
\begin{aligned}
\mathcal{H} & =\sum_{i, \sigma} \varepsilon_{\sigma} c_{i \sigma}^{\dagger} c_{i \sigma}+\sum_{\langle i j\rangle, \sigma} t_{\sigma}\left(c_{i \sigma}^{\dagger} c_{j \sigma}+\text { H.c. }\right) \\
& +U \sum_{i} n_{i \uparrow} n_{i \downarrow}+v_{0} \sum_{i}\left(c_{i \uparrow}^{\dagger} c_{i \downarrow}+\text { H.c. }\right) \\
& +v_{\uparrow \downarrow} \sum_{\langle i j\rangle}\left(c_{i \uparrow}^{\dagger} c_{j \downarrow}+\text { H.c. }\right) \\
& +v_{\downarrow \uparrow} \sum_{\langle i j\rangle}\left(c_{i \downarrow}^{\dagger} c_{j \uparrow}+\text { H.c. }\right)
\end{aligned}
$$

Here $\langle i j\rangle$ indicates that $i$ and $j$ are nearest-neighbor sites. The fermionic operators $c_{i \sigma}^{(\dagger)}$ annihilate (create) an electron on the spin $\sigma$ Wannier orbital of the lattice 
site $\mathbf{R}_{i}$. The lattice has a total number of $N$ sites, and $n_{j \sigma}=c_{j \sigma}^{\dagger} c_{j \sigma}$ is the particle number operator for site $j$ $(\sigma=\{\uparrow, \downarrow\}) . \varepsilon_{\sigma}$ denotes the on-site energy for each orbital, $t_{\sigma}$ are the intraband hopping amplitudes, $U$ is the local interorbital Coulomb interaction strength, and $v_{\gamma}$ are the interband hybridization amplitudes, where $\gamma=0$ for on-site hybridization and $\gamma=\{\uparrow \downarrow, \downarrow \uparrow\}$ for intersite hybridization. The EFKM is recovered from Eq. (11) by setting $v_{\gamma}=0$. In this limit, the model has a continuous $\mathrm{U}(1)$ symmetry, which is removed by the inclusion of an explicit hybridization. The discrete symmetries that remain for the more general HEFKM are spatial inversion and time-reversal invariance.

The pseudospin language of Eq. (11) unveils the similarity of $\mathcal{H}$ with other generic many-body Hamiltonians. The EFKM $\left(v_{\gamma}=0\right)$ becomes an asymmetric Hubbard model, i.e., a single band model for electrons with a spin-dependent dispersion. We will still use the name "Falicov-Kimball model" to indicate that the pseudospin degree of freedom represents a physical orbital degree of freedom. From now on, we will consider that $\mathcal{H}$ is defined on a square lattice and $\left\langle n_{i \uparrow}+n_{i \downarrow}\right\rangle=1$ (half-filled band case). We will also restrict to zero temperature and measure all energies in units of $t_{\uparrow}=1$. Finally, we will assume that the Wannier functions of $c$ and $f$ orbitals, $\phi_{\uparrow}\left(\mathbf{r}-\mathbf{R}_{i}\right)$ and $\phi_{\downarrow}\left(\mathbf{r}-\mathbf{R}_{i}\right)$, are real.

\section{ORDER PARAMETERS}

In the rest of the paper we will refer to the pseudospin simply as "spin." The spin representation used in Eq. (11) unveils the $\mathrm{SU}(2)$ structure of this internal degree of freedom. This degree of freedom is the only one that survives at low energies in the large $U /\left|t_{\sigma}\right|$ limit. Consequently, the three different local or real-space order parameters correspond to the three components of the local spin variable,

$$
\mathcal{S}_{j}=\frac{1}{2} \sum_{\sigma, \sigma^{\prime}} c_{j \sigma}^{\dagger} \boldsymbol{\sigma}_{\sigma \sigma^{\prime}} c_{j \sigma^{\prime}}
$$

where $\boldsymbol{\sigma}$ is the vector of the Pauli matrices. More complicated (or higher order) real-space order parameters involve products of spin operators in more than one unit cell.

A real space modulation of $\left\langle\mathcal{S}_{j}^{z}\right\rangle$ leads to orbital ordering. Here we will only consider the ordering wave vector $\mathbf{Q}=(\pi, \pi)$ that leads to staggered orbital ordering (SOO) because the effective interaction is antiferromagnetic between nearest-neighbors and the lattice under consideration is bipartite. The corresponding order parameter is

$$
\delta_{\mathrm{SOO}}=\sum_{j} e^{i \mathbf{Q} \cdot \mathbf{R}_{j}}\left\langle\mathcal{S}_{j}^{z}\right\rangle
$$

If the two orbitals have opposite parity, a nonzero $\left\langle\mathcal{S}_{j}^{x}\right\rangle$ implies the presence of a spontaneous local electric polarization that turns out to be uniform for the HEFKM.
This is the EFE that was found in previous works for particular limits of the HEFKM $\underline{14-16}$ The uniform electric polarization is given by

$$
\langle\mathcal{P}\rangle=\mathbf{p} \sum_{j}\left\langle\mathcal{S}_{j}^{x}\right\rangle
$$

with the interband dipole matrix element

$$
\mathbf{p}=2 e \int d^{3} r \phi_{\uparrow}(\mathbf{r}) \mathbf{r} \phi_{\downarrow}(\mathbf{r}),
$$

where $e$ is the electron charge. This phase breaks the spatial inversion symmetry of $\mathcal{H}$.

If the two orbitals have the same parity (for instance $s$ and $d$ orbitals), a nonzero modulation of $\left\langle\mathcal{S}_{j}^{x}\right\rangle$ corresponds to an electric quadrupole density wave (EQDW) as long as the tensor

$$
q_{\sigma \sigma^{\prime}}^{\nu \nu^{\prime}}=e \int d^{3} r \phi_{\sigma}(\mathbf{r}) r_{\nu} r_{\nu^{\prime}} \phi_{\sigma^{\prime}}(\mathbf{r})
$$

is nonzero for $\sigma^{\prime}=\bar{\sigma} \equiv-\sigma\left(\nu, \nu^{\prime}=\{x, y, z\}\right)$. In case the tensor $\mathbf{q}_{\sigma \bar{\sigma}}$ [Eq. (6)] vanishes, one has to look for the lowest order electric multipole that has a nonzero matrix element between the orbitals $\phi_{\uparrow}(\mathbf{r})$ and $\phi_{\downarrow}(\mathbf{r})$. In the rest of this paper, we will assume that the tensor $\mathbf{q}_{\sigma \bar{\sigma}}$ does not vanish. In second quantization, the local electric quadrupole tensor on the unit cell $j$ is given by the operator,

$$
\mathcal{Q}_{j}=\mathbf{q}_{\uparrow \uparrow} n_{j \uparrow}+\mathbf{q}_{\downarrow \downarrow} n_{j \downarrow}+2 \mathbf{q}_{\uparrow \downarrow} S_{j}^{x} .
$$

We note that $\mathbf{q}_{\uparrow \downarrow}=\mathbf{q}_{\downarrow \uparrow}$. The corresponding quadrupolar order parameter in momentum space is given by

$$
\left\langle\mathcal{Q}_{\mathbf{k}}\right\rangle=\sum_{j} e^{i \mathbf{k} \cdot \mathbf{R}_{j}}\left\langle\mathcal{Q}_{j}\right\rangle
$$

Again, for the Hamiltonian under consideration, the wave vector of the electric quadrupolar ordering is $\mathbf{k}=\mathbf{Q}$. Equation (77) implies that a nonzero modulation of the $x$ spin component, $\left\langle\mathcal{S}_{j}^{x}\right\rangle$, corresponds to an EQDW. While translational symmetry is broken in this phase, timereversal and spatial inversion symmetries are conserved. We note that the first two terms of Eq. (7) imply that orbital ordering will also lead to an EQDW. However, as it is also clear from Eq. (77), the quadrupolar tensors associated with the ordering along the $z$ and $x$ axes are different. The quadrupolar electric moment that is modulated under staggered orbital ordering (staggered $z$ component) corresponds to a linear combination of the tensors $\mathbf{q}_{\uparrow \uparrow}$ and $\mathbf{q}_{\downarrow \downarrow}$. On the other hand, the staggered ordering of the $x$-spin component involves a modulation of a quadrupolar electric tensor proportional to $\mathbf{q}_{\uparrow \downarrow}$ (hybridization-induced quadrupolar electric moment). In order to simplify the notation, we will use "EQDW" to denote the staggered ordering of the $x$-spin component and SOO for the the staggered ordering of the $z$-spin component. 
Finally, a nonzero $\left\langle\mathcal{S}_{j}^{y}\right\rangle$ implies the spontaneous emergence of a current-density distribution between the two orbitals of the unit cell $j: 21,27$ This can be easily verified if we neglect the overlap between orbitals that belong to different unit cells. In that case, the current-density operator at a point $\mathbf{r}$ near $\mathbf{R}_{j}$ is given by

$$
\mathbf{j}_{j}(\mathbf{r})=\frac{\hbar}{m_{e}} S_{j}^{y} \sum_{\sigma} \sigma \phi_{\bar{\sigma}}\left(\mathbf{r}-\mathbf{R}_{j}\right) \nabla_{\mathbf{r}} \phi_{\sigma}\left(\mathbf{r}-\mathbf{R}_{j}\right),
$$

where $m_{e}$ is the electron mass and the prefactor $\sigma$ takes the value +1 for $\uparrow$ and -1 for $\downarrow$. We note that this current density flows between the two orbitals of the same unit cell (these are atomic currents when the two orbitals belong to the same ion), in contrast to the orbital currents found in Ref. 28 that flow between different unit cells. This CHP breaks time-reversal symmetry and the physical order parameter is the lowest order nonzero multipole of the current-density distribution given by Eq. (9). The chiral ordering is staggered for orbitals with the same parity and uniform for orbitals with opposite parity. For instance, if we are considering two $p$ orbitals, the staggered chiral ordering of the $y$-spin component corresponds to orbital antiferromagnetism, because the current distribution given by Eq. (9) generates a net magnetic dipole moment. Since we will consider the general case of any arbitrary pair of orbitals, we will use "CHP" to denote the uniform ordering of the $y$-spin component (same parity orbitals) and "staggered chiral phase" (SCHP) to denote the staggered ordering.

\section{STRONG-COUPLING REGIME}

For the case $\left|t_{\sigma}\right|,\left|v_{\gamma}\right| \ll U$, we can perform a large- $U$ expansion, thereby reducing the HEFKM [Eq. (1)] to an effective strong-coupling Hamiltonian, $\mathcal{H}^{\mathrm{sc}}$, that reproduces the low-energy spectrum of the original model.

A large on-site Coulomb interaction splits the spectrum of the HEFKM Hamiltonian into high- and lowenergy parts. For $t_{\sigma}=v_{\gamma}=0$, the lowest-energy subspace is generated by the $2^{N}$ states that have one electron per site. The high-energy subspaces are separated by energy gaps equal to $U n_{d}$, where $n_{d}$ is the number of double occupied sites. For nonzero $t_{\sigma}$ and $v_{\gamma}$, the electrons are no longer completely localized at their ions, i.e., an electron can gain kinetic energy by visiting virtually a neighboring site. Since we are considering the half-filled band case (one particle per site), the low-energy effective model becomes a spin Hamiltonian $\mathcal{H}^{\mathrm{sc}}$. The expression for $\mathcal{H}^{\text {sc }}$ up to second order in the kinetic-energy terms is

$$
\begin{aligned}
\mathcal{H}^{\mathrm{sc}} & =\sum_{\langle i j\rangle}\left(J_{x x} \mathcal{S}_{i}^{x} \mathcal{S}_{j}^{x}+J_{y y} \mathcal{S}_{i}^{y} \mathcal{S}_{j}^{y}+J_{z z} \mathcal{S}_{i}^{z} \mathcal{S}_{j}^{z}\right) \\
& +\sum_{\langle i j\rangle}\left(J_{x z} \mathcal{S}_{i}^{x} \mathcal{S}_{j}^{z}+J_{z x} \mathcal{S}_{i}^{z} \mathcal{S}_{j}^{x}+C\right) \\
& +\sum_{i}\left(B \mathcal{S}_{i}^{z}+2 v_{0} \mathcal{S}_{i}^{x}\right)
\end{aligned}
$$

where

$$
\begin{aligned}
J_{x x} & =\frac{4}{U}\left(t_{\uparrow} t_{\downarrow}+v_{\uparrow \downarrow} v_{\downarrow \uparrow}\right), \\
J_{y y} & =\frac{4}{U}\left(t_{\uparrow} t_{\downarrow}-v_{\uparrow \downarrow} v_{\downarrow \uparrow}\right), \\
J_{z z} & =\frac{2}{U}\left(t_{\uparrow}^{2}+t_{\downarrow}^{2}-v_{\uparrow \downarrow}^{2}-v_{\downarrow \uparrow}^{2}\right), \\
J_{x z} & =\frac{4}{U}\left(t_{\uparrow} v_{\downarrow \uparrow}-t_{\downarrow} v_{\uparrow \downarrow}\right), \\
J_{z x} & =\frac{4}{U}\left(t_{\uparrow} v_{\uparrow \downarrow}-t_{\downarrow} v_{\downarrow \uparrow}\right), \\
C & =\varepsilon_{\uparrow}+\varepsilon_{\downarrow}-\frac{1}{2 U}\left(t_{\uparrow}^{2}+t_{\downarrow}^{2}+v_{\uparrow \downarrow}^{2}+v_{\downarrow \uparrow}^{2}\right), \\
B & =\varepsilon_{\uparrow}-\varepsilon_{\downarrow} .
\end{aligned}
$$

It is well known that the half-filled isotropic Hubbard model can be mapped on an effective Heisenberg model in the limit of a large Coulomb repulsion. For the more general EFKM, the intraband hopping amplitudes and the different on-site potentials lead to an effective XXZ model in a magnetic field $B$ along the $z$ axis. $B$ is simply the energy difference between the two orbitals: $\frac{15}{\underline{K}}$ As expected, the interband hybridization of the HEFKM generates anisotropic terms that explicitly break the U(1) invariance under uniform spin rotations about the $z$ axis. While the intersite hybridization leads to anisotropic exchange terms, the on-site hybridization leads to a Zeeman coupling to a uniform field along the $x$ axis.

For low enough values of $B$ and no interband hybridization, the ground-state of $\mathcal{H}^{\text {sc }}$ exhibits SOO. The simple reason is that $J_{z z} \geq\left|J_{x x, y y}\right|$, i.e., the effective XXZ model is easy-axis $\stackrel{15}{\frac{15}{2}}$ If $J_{z z}$ is significantly larger than $\left|J_{x x, y y}\right|$, the SOO remains robust when the interband hybridization is included. Clearly, there exists a critical value of $B$ that leads to a spin-flop transition to an ordered phase in the $X Y$ plane with a uniform component along the $z$ axis (canted $X Y$ phase). In absence of interband hybridization, the $\mathrm{U}(1)$ invariance of the EFKM implies that spin component perpendicular to the applied field can point along any direction of the $X Y$ plane. In other words, there is a continuous ground-state degeneracy that includes the EQDW (EFE) and SCHP (CHP) for orbitals with the same (opposite) parity. In this case, the inclusion of interband hybridization is very relevant because it lifts the continuous degeneracy and stabilizes only one of the two possible Ising-type orderings (along the $x$ or $y$ spin direction).

For orbitals with opposite parity, we have $t_{\uparrow} t_{\downarrow}<0$, $v_{\downarrow \uparrow}=-v_{\uparrow \downarrow}$ and $v_{0}=0$. These relationships are derived from simple symmetry considerations. In this case $J_{x x}=-\frac{4}{U}\left(\left|t_{\uparrow} t_{\downarrow}\right|+v_{\uparrow \downarrow}^{2}\right), J_{y y}=-\frac{4}{U}\left(\left|t_{\uparrow} t_{\downarrow}\right|-v_{\uparrow \downarrow}^{2}\right)$, and $J_{z x}=-J_{x z}$. Since $J_{x x}, J_{y y}<0$ and $\left|J_{x x}\right|>\left|J_{y y}\right|$, the energy is minimized by a ferromagnetic alignment of the spins along the $x$ direction that corresponds to an EFE phase. Since this was previously shown in Ref. 15, from now on we will concentrate on the case of equal parity orbitals. In this case, we have $t_{\uparrow} t_{\downarrow}>0, v_{\downarrow \uparrow}=v_{\uparrow \downarrow}$, and $v_{0}$ can be nonzero if the two orbitals belong to different ions. 
Then $J_{x x}>J_{y y}$ and $J_{z x}=J_{x z}$. Since $J_{x x}>J_{y y}>0$, $v_{\uparrow \downarrow}$ favors staggered Ising-type ordering along the $x$ direction, while $v_{0}$ favors a uniform polarization along the $x$ direction and, consequently, a staggered Ising-type ordering along the $y$ direction. In other words, the interband hybridization can stabilize an EQDW or a SCHP depending on the ratio between the on-site and intersite hybridization amplitudes.

We introduce now the mean-field variational states and the corresponding energies for the three order parameters that we introduced in the previous section.

(i) SOO. This phase has a staggered spin component along the $z$-direction, and a uniform component along the $x$ direction that can be induced by the on-site hybridization term $v_{0}$,

$$
\begin{aligned}
& \left\langle\mathcal{S}_{j}\right\rangle_{\theta_{1}}=S\left(\sin \theta_{1}, 0, \cos \theta_{1} e^{i \mathbf{Q R} \mathbf{R}_{j}}\right) \\
& E_{0}^{\mathrm{SOO}}=-D N S^{2} J_{z z} \cos ^{2} \theta_{1}+D N S^{2} J_{x x} \sin ^{2} \theta_{1} \\
& \quad+2 v_{0} S N \sin \theta_{1}+D N C .
\end{aligned}
$$

(ii) SCHP. This phase has a staggered spin component along the $y$ direction and uniform polarizations along the $x$ and $z$ directions,

$$
\begin{aligned}
\left\langle\mathcal{S}_{j}\right\rangle_{\theta_{1}, \theta_{2}} & =S\left(\sin \theta_{1} \cos \theta_{2}, e^{i \mathbf{Q R} \mathbf{R}_{j}} \sin \theta_{1} \sin \theta_{2}, \cos \theta_{1}\right) \\
E_{0}^{\mathrm{SCHP}} & =D N S^{2}\left(\left(J_{x x}+J_{y y}\right) \cos ^{2} \theta_{2}\left(1-\cos ^{2} \theta_{1}\right)-J_{y y}\right. \\
& \left.+\left(J_{y y}+J_{z z}\right) \cos ^{2} \theta_{1}+2 J_{x z} \sin \theta_{1} \cos \theta_{1} \cos \theta_{2}\right) \\
& +D N C+N S B \cos \theta_{1}+2 N S v_{0} \sin \theta_{1} \cos \theta_{2} .
\end{aligned}
$$

(iii) $E Q D W$. In this case the staggered spin component is aligned along the $x$ direction and there is a uniform component along the $z$ direction induced by $B$ (the $y$ component vanishes),

$$
\begin{aligned}
\left\langle\mathcal{S}_{j}\right\rangle_{\theta_{1}} & =S\left(e^{i \mathbf{Q R} \mathbf{R}_{j}} \sin \theta_{1}, 0, \cos \theta_{1}\right) \\
E_{0}^{\mathrm{EQDW}} & =D N C+N B S \cos \theta_{1}-D N J_{x x} S^{2} \sin ^{2} \theta_{1} \\
& +D N J_{z z} S^{2} \cos ^{2} \theta_{1} .
\end{aligned}
$$

In all cases we have $D=2, \mathbf{Q}=(\pi, \pi)$, and $S=1 / 2$. By minimizing the respective energies with respect to $\theta_{1}$ and $\theta_{2}$, we determine the quantum phase diagram as a function of the band-structure parameters.

Figure 1(a) shows the effect of a finite intersite hybridization amplitude, $v_{\uparrow \downarrow}$, on the quantum phase diagram of the EFKM. The intersite hybridization stabilizes the EQDW relative to the SCHP. On the other hand, the EQDW is also favored relative to the SOO because $v_{\uparrow \downarrow}$ decreases the value of $J_{z z}$ and simultaneously increases the value of $J_{x x}$ [see Eqs. (11) and (13)]. Figure 11(b) illustrates the effect of a finite on-site hybridization. In this case, the SCHP is favored relative to the EQDW. In contrast to $v_{\uparrow \downarrow}, v_{0}$ does not change the transition point between the SCHP and the SOO. The simple reason is that $v_{0}$ does not affect the exchange constants. It just generates an effective pseudomagnetic field along the $x$
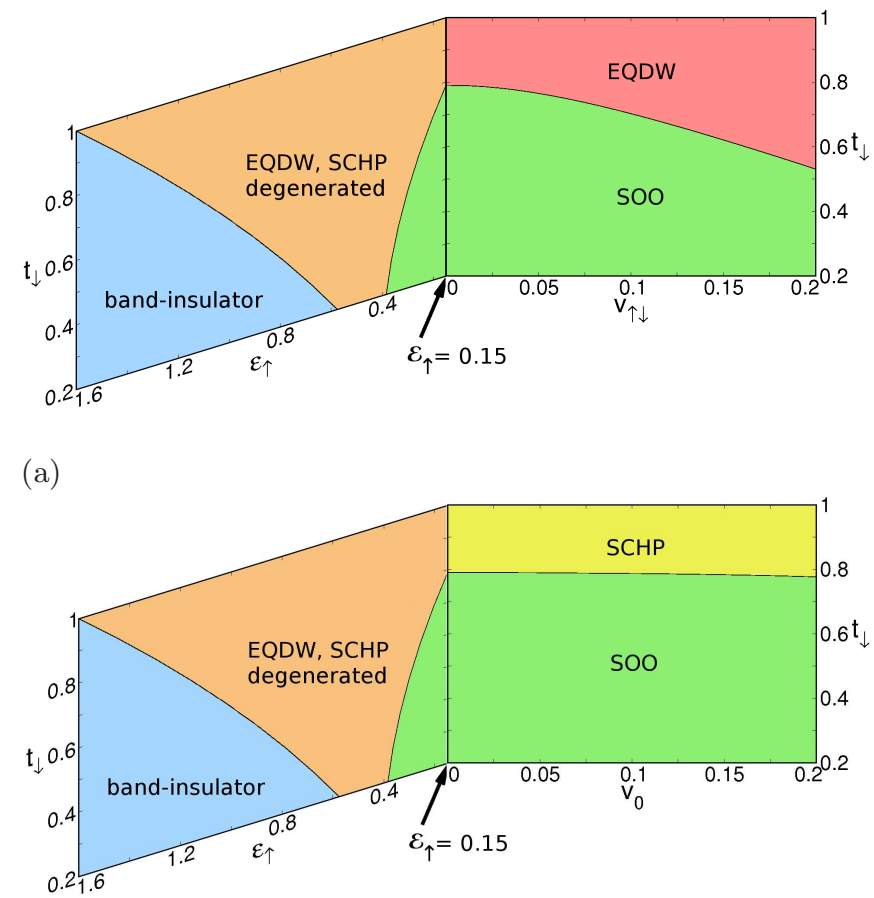

(b)

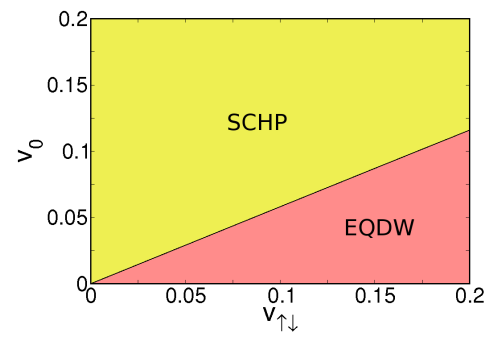

(c)

FIG. 1: (Color online) Ground-state phase diagram of the $2 \mathrm{D}$ EFKM in the strong-coupling regime. Band-structure parameters are $\varepsilon_{\downarrow}=0.0, t_{\uparrow}=1.0$, and $U=10$. Left-hand side diagrams [in panels (a) and (b)] give results for the nonhybridized EFKM $\left(v_{0}=0\right.$ and $\left.v_{\uparrow \downarrow}=0\right)$, while right-hand side diagrams show the dependence on the (a) intersite hybridization $v_{\uparrow \downarrow}$ and (b) on-site hybridization $v_{0}$ for $\varepsilon_{\uparrow}=0.15$. Panel (c) gives the stability region of the staggered chiral phase and the electric quadrupole density wave in dependence on $v_{0}$ and $v_{\uparrow \downarrow}$ for $\varepsilon_{\uparrow}=0.5, t_{\downarrow}=0.5$.

axis that leads to a finite canting angle in both phases. Figure 1(c) shows the phase diagram as a function of $v_{0}$ and $v_{\uparrow \downarrow}$ for a large enough value of $B=0.5$ and same parity orbitals. Again, we can see that a finite on-site hybridization $v_{0}$ strengthens the SCHP, while the intersite hybridization $v_{\uparrow \downarrow}$ stabilizes the EQDW. Within our simple mean-field approximation the boundary between these two phases is a straight line. 


\section{WEAK-COUPLING REGIME}

It has been shown in Ref. 18 that the mean-field ground-state phase diagram of the 2D EFKM agrees almost perfectly with the one obtained by a constrained path Monte Carlo technique, even in the intermediate coupling regime. This agreement motivates us to perform a Hartree-Fock decoupling of the HEFKM to explore the quantum phase diagram for small $U /\left|t_{\sigma}\right|$.

The weak-coupling analysis requires to express the relevant order parameters in momentum space. In particular, the Fourier components of $\left\langle\mathcal{S}_{j}^{x}\right\rangle$ and $\left\langle\mathcal{S}_{j}^{y}\right\rangle$ can be represented as a complex number,

$$
\begin{aligned}
\Delta_{\mathbf{Q}} & =\left|\Delta_{\mathbf{Q}}\right| e^{i \varphi}=\frac{U}{N} \sum_{\mathbf{k}}\left\langle c_{\mathbf{k}+\mathbf{Q} \uparrow}^{\dagger} c_{\mathbf{k} \downarrow}\right\rangle \\
& =\frac{U}{N} \sum_{j} e^{i \mathbf{Q R}_{j}}\left(\left\langle\mathcal{S}_{j}^{x}\right\rangle+i\left\langle\mathcal{S}_{j}^{y}\right\rangle\right) \\
& =\frac{U}{\sqrt{N}}\left(\left\langle\mathcal{S}_{\mathbf{Q}}^{x}\right\rangle+i\left\langle\mathcal{S}_{\mathbf{Q}}^{y}\right\rangle\right),
\end{aligned}
$$

where

$$
\begin{aligned}
c_{\mathbf{k} \sigma}^{\dagger} & =\frac{1}{\sqrt{N}} \sum_{j} e^{i \mathbf{k} \cdot \mathbf{R}_{j}} c_{j \sigma}^{\dagger}, \\
\mathcal{S}_{\mathbf{k}}^{\nu} & =\frac{1}{\sqrt{N}} \sum_{j} e^{i \mathbf{k} \cdot \mathbf{R}_{j}} \mathcal{S}_{j}^{\nu} .
\end{aligned}
$$

The ordering wave vector $\mathbf{Q}$ determines the modulation of the real-space order parameter. According to our strong-coupling analysis, we have $\mathbf{Q}=(\pi, \pi)$ for orbitals with the same parity and $\mathbf{Q}=(0,0)$ for orbitals with opposite parity. Again, the U(1) invariance of the EFKM $\left(v_{\gamma}=0\right)$ implies that the energy does not depend on $\varphi$. Consequently, there is an infinite number of groundstates with $\Delta_{\mathbf{Q}} \neq 0$ that results from the spontaneous U(1) symmetry breaking of the EFKM (excitonic condensate). A finite interband hybridization $\left(v_{\gamma} \neq 0\right)$ removes the continuous $\mathrm{U}(1)$ symmetry and lifts the $\varphi$ degeneracy of the HEFKM ground-state.

For orbitals with opposite parity, the hybridization in momentum space takes the form $v_{\mathbf{k}}=2 i v_{\uparrow \downarrow}\left(\sin k_{x}+\right.$ $\sin k_{y}$ ) and the EFE state has a lower energy than the CHP. This is in agreement with the result for the FKM extended by a (small) intersite hybridization in Ref. 14 . Therefore, from now on we will focus only on the equal parity case. The Hartree-Fock decoupling suggested by Eq. (24) gives

$$
\begin{aligned}
\mathcal{H}^{\mathrm{wc}} & =\sum_{\mathbf{k}, \sigma} \bar{\varepsilon}_{\mathbf{k} \sigma} c_{\mathbf{k} \sigma}^{\dagger} c_{\mathbf{k} \sigma}+\sum_{\mathbf{k}, \sigma} v_{\mathbf{k}} c_{\mathbf{k} \sigma}^{\dagger} c_{\mathbf{k}-\sigma} \\
& -\sum_{\mathbf{k}} \Delta_{\mathbf{Q}} c_{\mathbf{k} \downarrow}^{\dagger} c_{\mathbf{k}+\mathbf{Q} \uparrow}-\sum_{\mathbf{k}} \Delta_{\mathbf{Q}}^{*} c_{\mathbf{k}+\mathbf{Q} \uparrow}^{\dagger} c_{\mathbf{k} \downarrow}
\end{aligned}
$$

with

$$
\begin{aligned}
\bar{\varepsilon}_{\mathbf{k} \sigma} & =\varepsilon_{\sigma}+U n_{-\sigma}+2 t_{\sigma}\left(\cos k_{x}+\cos k_{y}\right), \\
v_{\mathbf{k}} & =v_{0}+2 v_{\uparrow \downarrow}\left(\cos k_{x}+\cos k_{y}\right), \\
n_{\sigma} & =\frac{1}{N} \sum_{\mathbf{k}}\left\langle c_{\mathbf{k} \sigma}^{\dagger} c_{\mathbf{k} \sigma}\right\rangle, \\
\Delta_{\mathbf{Q}} & =\frac{U}{N} \sum_{\mathbf{k}}\left\langle c_{\mathbf{k}+\mathbf{Q} \uparrow}^{\dagger} c_{\mathbf{k} \downarrow}\right\rangle, \\
\Delta_{\mathbf{Q}}^{*} & =\frac{U}{N} \sum_{\mathbf{k}}\left\langle c_{\mathbf{k} \downarrow}^{\dagger} c_{\mathbf{k}+\mathbf{Q} \uparrow}\right\rangle .
\end{aligned}
$$

The mean-field Hamiltonian (29) can be easily diagonalized by the canonical transformation 29

$$
\mathcal{C}_{\mathbf{k}, m}=u_{\mathbf{k}, m} c_{\mathbf{k} \uparrow}+v_{\mathbf{k}, m} c_{\mathbf{k} \downarrow}+\tilde{u}_{\mathbf{k}, m} c_{\mathbf{k}+\mathbf{Q} \uparrow}+\tilde{v}_{\mathbf{k}, m} c_{\mathbf{k}+\mathbf{Q} \downarrow},
$$

where $m=1,2,3,4$. The coefficients are solutions of the associated Bogoliubov de Gennes equations, $\mathcal{H}_{\mathbf{k}}^{\mathrm{wc}} \Psi_{\mathbf{k}, m}=$ $E_{\mathbf{k}, m} \Psi_{\mathbf{k}, m}$, with

$$
\mathcal{H}_{\mathbf{k}}^{\mathrm{wc}}=\left(\begin{array}{cccc}
\bar{\varepsilon}_{\mathbf{k} \uparrow} & v_{\mathbf{k}} & 0 & -\Delta_{\mathbf{Q}}^{*} \\
v_{\mathbf{k}} & \bar{\varepsilon}_{\mathbf{k} \downarrow} & -\Delta_{\mathbf{Q}} & 0 \\
0 & -\Delta_{\mathbf{Q}}^{*} & \bar{\varepsilon}_{\mathbf{k}+\mathbf{Q} \uparrow} & v_{\mathbf{k}+\mathbf{Q}} \\
-\Delta_{\mathbf{Q}} & 0 & v_{\mathbf{k}+\mathbf{Q}} & \bar{\varepsilon}_{\mathbf{k}+\mathbf{Q} \downarrow}
\end{array}\right)
$$

and $\Psi_{\mathbf{k}, m}=\left(u_{\mathbf{k}, m}, v_{\mathbf{k}, m}, \tilde{u}_{\mathbf{k}, m}, \tilde{v}_{\mathbf{k}, m}\right)^{T}$. The energy per site results as

$$
\frac{E_{0}^{\mathrm{wc}}}{N}=\frac{1}{N} \sum_{\mathbf{k}, m}{ }^{\prime} E_{\mathbf{k}, m} f\left(E_{\mathbf{k}, m}\right)-U n_{\uparrow} n_{\downarrow}+\frac{1}{U}\left|\Delta_{\mathbf{Q}}\right|^{2}
$$

where $f\left(E_{\mathbf{k}, m}\right)$ is the Fermi function containing the new quasiparticle energies $E_{\mathbf{k}, m}$ and the prime denotes that the $\mathbf{k}$ summation extends over the magnetic Brillouin zone only. The chemical potential $\mu$ is determined by the condition

$$
1=\frac{1}{N} \sum_{\mathbf{k}, m}^{\prime} f\left(E_{\mathbf{k}, m}\right) .
$$

Next we consider the mean-field decoupling that leads to SOO. In this case, we introduce the possibility of a periodic modulation in the electronic density with independent amplitudes for each spin polarization,

$$
\left\langle n_{i \sigma}\right\rangle=n_{\sigma}+\delta_{\sigma} \cos \left(\mathbf{Q R} \mathbf{R}_{i},\right.
$$

with

$$
\delta_{\sigma}=\frac{1}{N} \sum_{\mathbf{k}}\left\langle c_{\mathbf{k} \sigma}^{\dagger} c_{\mathbf{k}+\mathbf{Q} \sigma}\right\rangle .
$$

The associated Bogoliubov de Gennes equations are $\mathcal{H}_{\mathbf{k}}^{\mathrm{SOO}} \Psi_{\mathbf{k}, m}=E_{\mathbf{k}, m}^{\mathrm{SOO}} \Psi_{\mathbf{k}, m}$, with

$$
\mathcal{H}_{\mathbf{k}}^{\mathrm{SOO}}=\left(\begin{array}{cccc}
\bar{\varepsilon}_{\mathbf{k} \uparrow} & v_{\mathbf{k}} & U \delta_{\uparrow} & 0 \\
v_{\mathbf{k}} & \bar{\varepsilon}_{\mathbf{k} \downarrow} & 0 & U \delta_{\downarrow} \\
U \delta_{\uparrow} & 0 & \bar{\varepsilon}_{\mathbf{k}+\mathbf{Q} \uparrow} & v_{\mathbf{k}+\mathbf{Q}} \\
0 & U \delta_{\downarrow} & v_{\mathbf{k}+\mathbf{Q}} & \bar{\varepsilon}_{\mathbf{k}+\mathbf{Q} \downarrow}
\end{array}\right) .
$$


The SOO order parameter becomes

$$
\delta_{\mathrm{SOO}}=\frac{\delta_{\uparrow}-\delta_{\downarrow}}{2}
$$

For asymmetric bands, $t_{\uparrow} \neq t_{\downarrow}$, the presence of a nonzero SOO leads to a secondary charge-density-wave (CDW) order, whose order parameter is given by

$$
\delta_{\mathrm{CDW}}=\frac{\delta_{\uparrow}+\delta_{\downarrow}}{2} .
$$

This secondary CDW provides a simple way of detecting the SOO in real materials. The mean-field energy per site that results from such a kind of SOO is

$$
\frac{E_{0}^{\mathrm{SOO}}}{N}=\frac{1}{N} \sum_{\mathbf{k}, m}{ }^{\prime} E_{\mathbf{k}, m}^{\mathrm{SOO}} f\left(E_{\mathbf{k}, m}^{\mathrm{SOO}}\right)-U n_{\uparrow} n_{\downarrow}-U \delta_{\downarrow} \delta_{\uparrow} .
$$

By solving the self-consistency Eqs. (33) and (40), and comparing the corresponding mean-field energies given by Eqs. (37) and (44), we compute the ground-state phase diagram.

Figure 2 is the weak-coupling counterpart of Figure 1 Like for the strong-coupling regime, Fig. 2(a) shows that an increasing value of $v_{\uparrow \downarrow}$ narrows the SOO phase while the region of the EQDW phase is enlarged. On the other hand, the on-site hybridization $v_{0}$ favors the SCHP relative to the EQDW and it does not have a noticeable effect on the transition line between the SOO and the SCHP [see Fig. 2(b)]. This also coincides with the strongcoupling results. Figure 2(c) shows the stability regions of the EQDW and SCHP as a function of the hybridization amplitudes for a large enough $\left|\varepsilon_{\uparrow}-\varepsilon_{\downarrow}\right|=0.5$. Qualitatively, the result of our Hartree-Fock approach is similar to the one obtained from the strong-coupling analysis [see Fig. 1(c)]. However, a more quantitative analysis shows that the area of stability for the SCHP is reduced relative to the strong-coupling result. A large Coulomb repulsion inhibits hopping processes and consequently reduces the influence of the intersite hybridization $v_{\uparrow \downarrow}$ relative to the effect of the on-site hybridization $v_{0}$.

\section{CONCLUSIONS}

The Hamiltonian that we considered in this work is a very simple extension of the Falicov-Kimball model. In spite of its simplicity, we have shown that this model leads to a very rich quantum phase diagram that contains all the possible local order parameters (three different components of the local spin $\mathcal{S}_{j}$ ) considered in Sec. III. The ordering wave vector $\mathbf{Q}$ is selected by the nesting property of the noninteracting Fermi surface in the weak-coupling limit and by the antiferromagnetic nature of the exchange interactions on a bipartite lattice in the strong-coupling limit. Most notably, the stability of the different broken symmetry states is very sensitive to

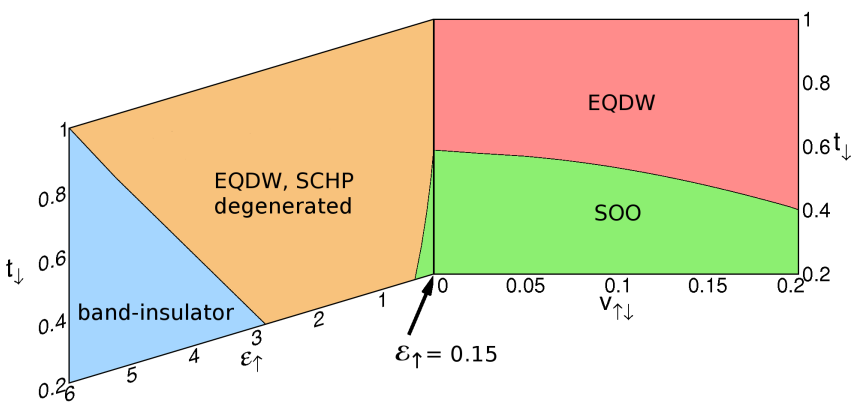

(a)

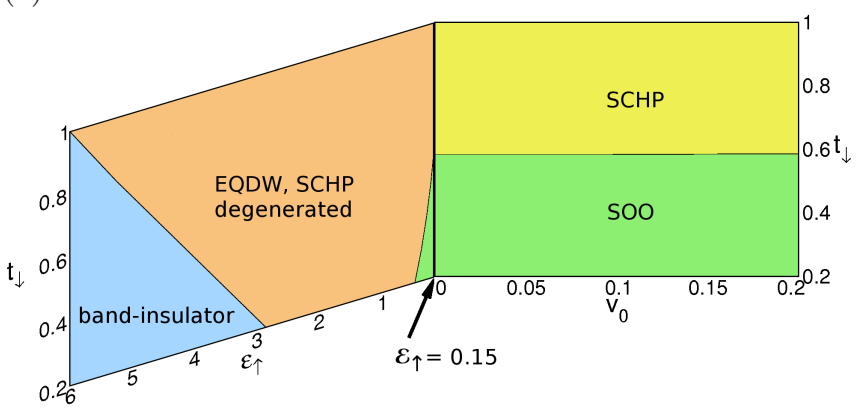

(b)

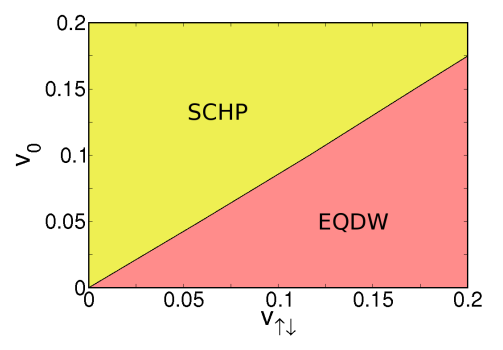

(c)

FIG. 2: (Color online) Ground-state phase diagram of the 2D EFKM in the weak-coupling regime. Band-structure parameters are $\varepsilon_{\downarrow}=0.0, t_{\uparrow}=1.0$, and $U=2$. Left-hand side diagrams [in panels (a) and (b)] give results for the nonhybridized EFKM $\left(v_{0}=0\right.$ and $\left.v_{\uparrow \downarrow}=0\right)$, while right-hand side diagrams show the dependence on the (a) intersite hybridization $v_{\uparrow \downarrow}$ and (b) on-site hybridization $v_{0}$ for $\varepsilon_{\uparrow}=0.15$. Panel (c) gives the stability region of the staggered chiral phase and the electric quadrupole density wave in dependence on $v_{0}$ and $v_{\uparrow \downarrow}$ for $\varepsilon_{\uparrow}=0.5, t_{\downarrow}=0.5$.

a few band-structure parameters. According to these results, it is necessary to have very accurate information about the band-structure properties near the Fermi energy to predict the correct ordered state. In particular, if the two orbitals have different angular momentum (like $s$ and $d$ orbitals), the SCHP may remain hidden to most of the experimental probes. The simple reason is that the spontaneous current-density distribution given by Eq. (9) has no net magnetic moment. Consequently, this phase can only be detected by using an experimental probe that couples to the lowest nonzero multipole of the currentdensity distribution. The SCHP becomes stable above 
a critical value of the on-site hybridization as long as the diagonal energy difference between the two orbitals $\left|\varepsilon_{c}-\varepsilon_{f}\right|$ is also larger than a minimal value.

Although all the calculations of this work were done for $D=2$, we do not expect any qualitative change for $D>2$. The obtained consistency between the weakand the strong-coupling approaches suggests that our results are robust. In particular, the absence of geometric frustration in the strong-coupling regime, whose weak-coupling counterpart is the nesting property of the Fermi surface, facilitates the search for the broken symmetry state that minimizes the energy for each set of Hamiltonian parameters. For orbitals with opposite parity under spatial inversion, we confirmed that the ferroelectric phase has always a lower energy than the chiral phase. For orbitals with the same parity, we found that the stabilization of the electric quadrupole density wave or the staggered chiral phase depends strongly on the dominant interband hybridization. The on-site hybridization, that is only allowed when the two orbitals belong to different ions, favors the staggered chiral phase, while a nearest-neighbor interband hybridization favors the electric quadrupole density wave.

\section{ACKNOWLEDGMENTS}

This work was supported by the Deutsche Forschungsgemeinschaft through SFB 652, B5, and by the NNSA of the U.S. DOE at the Los Alamos National Laboratory under Contract No. DE-AC52-06NA25396. B.Z. and H.F. are grateful for the hospitality provided at the Los Alamos National Laboratory.
1 L. M. Falicov and J. C. Kimball, Phys. Rev. Lett. 22, 997 (1969).

2 R. Ramirez, L. M. Falicov, and J. C. Kimball, Phys. Rev. B 2, 3383 (1970).

3 J. K. Freericks and V. Zlatić, Rev. Mod. Phys. 75, 1333 (2003).

4 C. Gruber and N. Macris, Helv. Phys. Acta 69, 850 (1996).

5 D. W. Pohl, Phys. Rev. B 15, 3855 (1977).

6 M. D. Nunez-Regueiro and M. Avignon, Phys. Rev. Lett. 55, 615 (1985).

7 J. K. Freericks, B. K. Nikolić, and P. Miller, Phys. Rev. B 64, 054511 (2001).

8 J. K. Freericks and T. P. Devereaux, Phys. Rev. B 64, 125110 (2001).

9 J. Mazzaferro, H. Ceva, and B. Alascio, Phys. Rev. B 22, 353 (1980).

10 A. V. Goltsev and G. Bruls, Phys. Rev. B 63, 155109 (2001).

11 S. H. Liu, Phys. Rev. B 64, 184108 (2001).

12 V. Subrahmanyam and M. Barma, J. Phys. C. 21, L19 (1988).

13 K. Kanda, K. Machida, and T. Matsubara, Solid State Commun. 19, 651 (1976).

14 T. Portengen, T. Östreich, and L. J. Sham, Phys. Rev. B 54, 17452 (1996).

15 C. D. Batista, Phys. Rev. Lett. 89, 166403 (2002).
16 C. D. Batista, J. E. Gubernatis, J. Bonča, and H. Q. Lin, Phys. Rev. Lett. 92, 187601 (2004).

17 P. Farkašovský, Phys. Rev. B 59, 9707 (1999).

18 P. Farkašovský, Phys. Rev. B 77, 155130 (2008).

19 N. F. Mott, Philos. Mag. 6, 287 (1961).

20 R. Knox, in Solid State Physics, edited by F. Seitz and D. Turnbull (Academic Press, New York, 1963), p. Suppl. 5 p. 100.

21 B. I. Halperin and T. M. Rice, Rev. Mod. Phys. 40, 755 (1968).

22 C. Schneider and G. Czycholl, Eur. Phys. J. B 64, 43 (2008).

23 D. Ihle, M. Pfafferott, E. Burovski, F. X. Bronold, and H. Fehske, Phys. Rev. B 78, 193103 (2008).

${ }^{24}$ P. M. R. Brydon, Phys. Rev. B 77, 045109 (2008).

25 B. Zenker, D. Ihle, F. X. Bronold, and H. Fehske, Phys. Rev. B 81, 115122 (2010).

${ }^{26}$ V.- N. Phan, K. W. Becker, and H. Fehske, Phys. Rev. B 81, in press (2010).

27 D. W. Buker, Phys. Rev. B 24, 5713 (1981).

${ }^{28}$ L. N. Bulaevskii, C. D. Batista, M. V. Mostovoy, and D. I. Khomskii, Phys. Rev. B 78, 024402 (2008).

29 P. M. R. Brydon, J.-X. Zhu, M. Gulácsi, and A. R. Bishop, Phys. Rev. B 72, 125122 (2005). 